\title{
Regularity of the effective diffusivity of random diffusion with respect to anisotropy coefficient
}

by

\author{
M. Cudna (Lublin) and T. Komorowski (Lublin and Warszawa) \\ Dedicated to Professor Jan Kisyński on the occasion of his 75th birthday
}

\begin{abstract}
We show that the effective diffusivity of a random diffusion with a drift is a continuous function of the drift coefficient. In fact, in the case of a homogeneous and isotropic random environment the function is $C^{\infty}$ smooth outside the origin. We provide a one-dimensional example which shows that the diffusivity coefficient need not be differentiable at 0 .
\end{abstract}

1. Introduction. A simple model of diffusion in a complicated medium is given by an Itô stochastic differential equation

$$
\begin{aligned}
d x(t) & =V(x(t) ; \omega) d t+\sigma(x(t) ; \omega) d w(t), \\
x(0) & =x_{0}
\end{aligned}
$$

(see e.g. [9]). Here $V(x ; \omega)=\frac{1}{2} \nabla_{x} \cdot a(x ; \omega), x \in \mathbb{R}^{d}$, where $a(x ; \omega)$ is a stationary, $d \times d$ matrix valued, random field given over a probability space $(\Omega, \mathcal{F}, \mathbb{P})$ and $\sigma(x ; \omega), x \in \mathbb{R}^{d}$, is the square root of the symmetric part of the field, $a^{\mathrm{S}}(x ; \omega)=\frac{1}{2}\left[a(x ; \omega)+a^{T}(x ; \omega)\right]$. The process $(w(t))_{t \geq 0}$ is a $d$-dimensional, standard Brownian motion independent of the field $a(\cdot)$. The symmetric part $\left(a^{\mathrm{S}}(x)\right)_{x \in \mathbb{R}^{d}}$ is assumed to be uniformly positive definite and sufficiently regular so that the solution of the stochastic differential equation exists; the precise assumptions are formulated in the next section. The generator of the random diffusion $(x(t))_{t \geq 0}$ is a second order differential operator in divergence form: $\mathcal{L}_{\omega} f=\frac{1}{2} \nabla_{x} \cdot\left(a(x ; \omega) \nabla_{x} f\right)$ for $f \in C_{0}^{2}\left(\mathbb{R}^{d}\right)$. We adopt the standard notation for usual function spaces: $C_{0}^{m}\left(\mathbb{R}^{d}\right)$ denotes the space of

2000 Mathematics Subject Classification: Primary 60F17, 35B27; Secondary 60G44.

Key words and phrases: random diffusion, self-diffusivity matrix.

The research of both authors was supported by Ministry of Science and Higher Education grant no N201 045 31. The research of T.K. has also been supported by EC FP6 Marie Curie ToK programme SPADE2, MTKD-CT-2004-014508 and Polish MNiSW SPB-M. 
all $C^{m}$-smooth, compactly supported functions, $C_{b}\left(\mathbb{R}^{d}\right)$ is the space of all bounded continuous functions, etc. It is well known from homogenization theory that the process $(x(t))_{t \geq 0}$ satisfies both the law of large numbers and the central limit theorem (see e.g. $[10,5]$ ), i.e. $x(t) / t$ tends a.s. to 0 as $t \rightarrow \infty$, while $x(t) / \sqrt{t}$ converges weakly to a zero mean normal vector with a non-degenerate diffusivity matrix $D=\left[D_{p q}\right]$. Unfortunately, the matrix is not given explicitly and its calculation is, in general, quite difficult.

Suppose now that an external force is added. As a result the particle acquires a drift. The equation describing the motion in a constant external force field is given by

$$
\begin{aligned}
d x(t) & =[V(x(t) ; \omega)+v] d t+\sigma(x(t) ; \omega) d w(t), \\
x(0) & =x_{0},
\end{aligned}
$$

where $v \in \mathbb{R}^{d}$ is a constant vector. One can show that then $x(t) / t \rightarrow v$ a.s. as $t \rightarrow \infty$. In addition, using a rather standard homogenization argument (see Theorem 2.1 below), one can prove that $(x(t)-v t) / \sqrt{t}$ tends to a zero mean normal vector with covariance matrix $D(v)=\left[D_{p q}(v)\right]$.

In the present paper we are concerned with smoothness of the covariance matrix with respect to the anisotropy parameter $v$. It has been shown in [6] that the function $v \mapsto D(v)$ is continuous at 0 . We prove (see Theorem 2.2 below) that in fact it is continuous for all $v \in \mathbb{R}^{d}$. Moreover, if the field $a(\cdot)$ is statistically isotropic (see Definition 2.5), then the diffusivity is an isotropic tensor in $v$ and $C^{\infty}$-smooth on $\mathbb{R}^{d} \backslash\{0\}$. As we explain in Section 6, this result is not entirely intuitively clear. Not getting too technical at this point, we only mention that a quite natural numerical scheme for computing the derivatives of the solution of the corresponding cell problem (see (3.8) below) does not imply the existence of the derivatives (see Remark 6.3). In fact, as shown in the one-dimensional example (see Section 5), the diffusivity coefficient $D(v)$ may not be differentiable at 0 .

2. Preliminaries and the statement of main theorems. Suppose that $(\Omega, \mathcal{F}, \mathbb{P})$ is a probability space. We shall denote by $\langle\cdot\rangle_{\mathbb{P}}$ the expectation with respect to $\mathbb{P}$. To describe the homogeneous random field $(a(x))_{x \in \mathbb{R}^{d}}$ it is convenient to assume that there exists an ergodic group $\left(\tau_{x}\right)_{x \in \mathbb{R}^{d}}$ of measure preserving transformations on $\Omega$, i.e. $\mathbb{P}\left(\tau_{x}(A)\right)=\mathbb{P}(A)$ for all $x \in \mathbb{R}^{d}$, and $A \in \mathcal{F}$ and $\tau_{x}(A)=A$ for all $x \in \mathbb{R}^{d}$ implies that $A$ is $\mathbb{P}$-trivial. We assume that the group is measurable and stochastically continuous, i.e. $(x, \omega) \mapsto$ $\mathbf{1}_{A}\left(\tau_{x} \omega\right)$ is jointly measurable for any $A \in \mathcal{F}$, and the unitary group $T_{x}$ : $L^{2}(\mathbb{P}) \rightarrow L^{2}(\mathbb{P}), x \in \mathbb{R}^{d}$, given by $T_{x} f(\omega):=f\left(\tau_{x} \omega\right)$ is strongly continuous. Denote by $\langle\cdot, \cdot\rangle_{\mathbb{P}},\|\cdot\|_{L^{2}(\mathbb{P})}$ the scalar product and the corresponding norm on $L^{2}(\mathbb{P})$. Let $D_{p}, p=1, \ldots, d$, be the $L^{2}$-infinitesimal generators of the 
group in the directions $\vec{e}_{p}:=(0, \ldots, 1, \ldots, 0), p=1, \ldots, d$. Let also $\nabla:=$ $\left(D_{1}, \ldots, D_{d}\right)$.

Let $a: \Omega \rightarrow \mathbb{R}^{d^{2}}$ be a random matrix for which there exists a deterministic constant $\lambda_{0}>0$ such that

$$
a(\omega) \xi \cdot \xi \geq \lambda_{0}|\xi|^{2}, \quad \forall \xi \in \mathbb{R}^{d} \text { and } \mathbb{P} \text {-a.s. } \omega .
$$

We assume furthermore that the entries of the matrix $a=\left[a_{p q}\right]$ satisfy

$$
\operatorname{ess} \sup \left\{\left|a_{p q}(\omega)\right|+\sum_{k=1}^{d}\left|D_{k} a_{p q}(\omega)\right|+\sum_{k, l=1}^{d}\left|D_{k l}^{2} a_{p q}(\omega)\right|\right\}<\infty
$$

for all $p, q=1, \ldots, d$. For a given random matrix $a$ we can define a homogeneous random field $a(x ; \omega):=a\left(\tau_{x} \omega\right),(\omega, x) \in \Omega \times \mathbb{R}^{d}$. Similarly, one can associate a random vector or scalar field with an arbitrary random vector or variable.

2.1. The statements of main results. We now give the precise statements of our main results. Their proofs are contained in the following sections.

Let $(x(t))_{t \geq 0}$ be the solution to (1.2). With no loss of generality we set $x_{0}=0$. We assume that the standard Brownian motion $(w(t))_{t \geq 0}$ appearing there is given over a certain probability space $(\Sigma, \mathcal{W}, \mathbb{Q})$. The trajectory process is defined over the product probability space $(\Omega \times \Sigma, \mathcal{F} \otimes \mathcal{W}, \mathbb{P} \otimes \mathbb{Q})$. In our first result we give the law of large numbers and the central limit theorem for the solutions of (1.2).

TheOREM 2.1. We have $x(t) / t \rightarrow v$ as $t \rightarrow \infty$, both a.s. and in $L^{1}$ with respect to the measure $\mathbb{P} \otimes \mathbb{Q}$. In addition, there exists a d-dimensional, zero mean Gaussian measure $\nu_{*}$ on $\mathbb{R}^{d}$ such that for any $f \in C_{b}\left(\mathbb{R}^{d}\right)$,

$$
\lim _{t \rightarrow \infty}\left\langle\left[E_{\mathbb{Q}} f\left(\frac{x(t)-v t}{\sqrt{t}}\right)-\int f d \nu_{*}\right]^{2}\right\rangle_{\mathbb{P}}=0 .
$$

Here $E_{\mathbb{Q}}$ and $\langle\cdot\rangle_{\mathbb{P}}$ denote the expectations with respect to $\mathbb{Q}$ and $\mathbb{P}$, respectively.

The quenched version of this result has already appeared in [6], where an analytic proof, using Harnack estimates for linear parabolic p.d.e-s, has been given. For completeness we present below (see Section 3) an argument that reduces the central limit theorem to certain properties of correctors, i.e. solutions of the resolvent equation (3.8), that can be verified when the generator is a bounded perturbation of a normal operator (see Appendix B below and also [4]).

Let $D(v)=\left[D_{p q}(v)\right]$ be the diffusivity matrix of $\nu_{*}$.

THEOREM 2.2. Suppose that the field of coefficients satisfies assumptions (2.1) and (2.2). Then the function $v \mapsto D(v)$ is continuous. 
The proof of this result is contained in Section 4.

2.2. Isotropic fields and $C^{\infty}$-smoothness of diffusivity. Let $O(d)$ denote the topological group of real orthonormal $d \times d$ matrices.

Definition 2.3. Suppose that $B: \mathbb{R}^{d} \rightarrow \mathbb{R}^{d^{2}}$ is a $d \times d$ matrix valued function. We say that the function is isotropic if

$$
B(g x)=g B(x) g^{T}, \quad x \in \mathbb{R}^{d}, g \in O(d) .
$$

We can think of $B(x)$ as a linear operator acting on $\mathbb{R}^{d}$. An isotropic, matrix valued function remains unchanged under the orthogonal change of coordinates $x^{\prime}:=g x, x \in \mathbb{R}^{d}$, where $g \in O(d)$. One can prove the following characterization of the second order symmetric isotropic tensors.

Proposition 2.4. Suppose that $B: \mathbb{R}^{d} \rightarrow \mathbb{R}^{d^{2}}$ is a symmetric matrix valued, isotropic function. Then there exist functions $f_{1}, f_{2}:(0, \infty) \rightarrow \mathbb{R}$ such that

$$
B(x)=f_{1}(|x|)(\operatorname{Id}-\widehat{x} \otimes \widehat{x})+f_{2}(|x|) \widehat{x} \otimes \widehat{x} .
$$

Here $\widehat{x} \otimes \widehat{x}:=\left[|x|^{-2} x_{p} x_{q}\right]$.

This is a well known result, especially in the physics and engineering literature; for completeness we recall its proof in Appendix A. One can also define a notion of statistical isotropy for a random matrix valued field.

DeFinition 2.5. We say that $a(\cdot)$ is statistically isotropic if for any $g \in$ $O(d)$ the law of the field $(a(g x))_{x \in \mathbb{R}^{d}}$ is the same as that of $\left(g a(x) g^{T}\right)_{x \in \mathbb{R}^{d}}$.

THEOREM 2.6. Suppose that in addition to assumptions (2.1) and (2.2) the field of coefficients is statistically isotropic. Then the function $v \mapsto D(v)$ is $C^{\infty}$ on $\mathbb{R}^{d} \backslash\{0\}$.

\section{The proof of Theorem 2.1}

3.1. Environment process, Dirichlet spaces and the law of large numbers. The material discussed in this section is standard in probabilistic homogenization theory. We shall refer a reader to either [7] or [8] for detailed proofs of the results cited here (see also [4]).

A convenient tool, frequently used in homogenization theory of random diffusions, is the so called environment process. It is an $\Omega$-valued stochastic process describing the environment from the vantage point of the diffusing particle $\zeta_{t}:=\tau_{x(t)}(\omega)$. It can be shown that the process is Markovian. To describe its generator define the following function spaces. For a non-negative integer $m$ let $C^{m}(\Omega)$ be the space of all random variables $F$ that have all partial derivatives $D_{1}^{m_{1}} \ldots D_{d}^{m_{d}} F, m_{1}+\cdots+m_{d}=m$, such that the corresponding random fields are continuous and deterministically bounded. Define also $C^{\infty}(\Omega):=\bigcap_{m \geq 0} C^{m}(\Omega)$. One can show that the measure $\mathbb{P}$ is invariant 
for the process considered. The transition semigroup extends to a strongly continuous semigroup on $L^{2}(\mathbb{P})$, and $C^{2}(\Omega)$ is a core of the $L^{2}(\mathbb{P})$-generator $\mathcal{L}^{(v)}$ of the process, given by the formula

$$
\mathcal{L}^{(v)} F(\omega)=\mathcal{L}^{(0)} F(\omega)+\sum_{p=1}^{d} v_{p} D_{p} F(\omega), \quad F \in C^{2}(\Omega),
$$

where

$$
\mathcal{L}^{(0)} F(\omega)=\frac{1}{2} \sum_{p, q=1}^{d} D_{p}\left(a_{p q} D_{q} F(\omega)\right), \quad F \in C^{2}(\Omega) .
$$

Suppose that $\mathcal{L}^{(v)} F=0$. Since

$$
0=-\left\langle\mathcal{L}^{(v)} F, F\right\rangle_{\mathbb{P}}=\frac{1}{2} \sum_{p, q=1}^{d}\left\langle a_{p q}^{\mathrm{s}} D_{p} F, D_{q} F\right\rangle_{\mathbb{P}} \geq \frac{\lambda_{0}}{2} \sum_{p=1}^{d}\left\|D_{p} F\right\|_{L^{2}(\mathbb{P})}^{2},
$$

the above implies that $\nabla F=0$. Ergodicity of the group $\left(\tau_{x}\right)_{x \in \mathbb{R}^{d}}$ of spatial shifts implies that $F \equiv$ const $\mathbb{P}$-a.s. This in turn shows that the only bounded functions that satisfy $\mathcal{L}^{(v)} F=0$ are constant $\mathbb{P}$-a.s. Thus, the measure $\mathbb{P}$ is ergodic for the process $\left(\zeta_{t}\right)_{t \geq 0}$. Equation (1.2) reads

$$
x(t)=\int_{0}^{t} c^{(v)}\left(\zeta_{s}\right) d s+M(t)
$$

where $c_{q}^{(v)}(\omega):=v_{q}+\frac{1}{2} \sum_{p=1}^{d} D_{p} a_{p q}(\omega)$ and

$$
M(t):=\int_{0}^{t} \sigma\left(\zeta_{s}\right) d w(s), \quad t \geq 0
$$

is a martingale of bounded quadratic variation. With the help of the ergodic theorem and the law of large numbers for martingales (see e.g. Theorem VII.9.2 of [2]), one can therefore conclude that $\lim _{t \rightarrow \infty} x(t) / t=\left\langle c^{(v)}\right\rangle_{\mathbb{P}}=v$ both a.s. and in $L^{1}$.

Let $L_{0}^{2}(\mathbb{P}):=\left[f \in L^{2}(\mathbb{P}): f \perp \mathbf{1}\right]$. Define the Hilbert space $H^{1}$ as the completion of $H_{0}^{1}$, the subspace of $L_{0}^{2}(\mathbb{P})$ consisting of those $F$ for which

$$
\|F\|_{1}^{2}:=\sum_{p=1}^{d}\left\|D_{p} F\right\|_{L^{2}(\mathbb{P})}^{2}<\infty
$$

in the norm given by (3.6). The corresponding scalar product is the unique extension of $\langle F, G\rangle_{1}:=\sum_{p=1}^{d}\left\langle D_{p} F, D_{p} G\right\rangle_{\mathbb{P}}, F, G \in H_{0}^{1}$. We denote by $H^{-1}$ the Hilbert space dual to $H^{1}$. Let $H_{0}^{-1} \subset L_{0}^{2}(\mathbb{P})$ consist of those $F \in L_{0}^{2}(\mathbb{P})$ for which $G \mapsto\langle F, G\rangle_{\mathbb{P}}, G \in H_{0}^{1}$, extends to a bounded linear functional $\iota(F) \in H^{-1}$ defined on the entire $H^{1}$. The mapping $\iota: H_{0}^{-1} \rightarrow H^{-1}$ is a 
linear embedding and

$$
\|\iota(F)\|_{-1}=\sup _{\|G\|_{1} \leq 1}\langle F, G\rangle_{\mathbb{P}}<\infty .
$$

We shall identify $H_{0}^{-1}$ with the range of $\iota$ and omit the notation for the operator.

Note that $D_{p}, p=1, \ldots, d$, extend to bounded linear operators from $H^{1}$ to $L^{2}(\mathbb{P})$. It can be checked directly that the operator $\Delta:=\sum_{p=1}^{d} D_{p}^{2}$ extends from $C^{2}(\Omega)$ to a unitary mapping between $H^{1}$ and $H^{-1}$.

3.2. Central limit theorem. In this section we prove that the laws of $((x(t)-v t) / \sqrt{t})_{t>0}$ converge weakly, as $t \rightarrow \infty$, to the law of a zero mean, Gaussian random vector. We start with the cell problem, i.e. the resolvent equation

$$
\left(\lambda-\mathcal{L}^{(v)}\right) \chi_{\lambda, v}^{(p)}=c_{p}
$$

where $\lambda>0$ and $c_{p}:=c_{p}^{(0)}, p=1, \ldots, d$. Observe that for any $F \in H_{0}^{1}$,

$$
\left\langle c_{p}, F\right\rangle_{\mathbb{P}}=-\frac{1}{2} \sum_{q=1}^{d}\left\langle a_{p q} D_{q} F\right\rangle_{\mathbb{P}}
$$

thus, by definition, each $c_{p}$ is in $H_{0}^{-1}$. Mulitplying both sides of (3.8) by $\chi_{\lambda, v}^{(p)}$, integrating over $\mathbb{P}$ and using coercivity (see $(2.1)$ ), we obtain

$$
\lambda\left\|\chi_{\lambda, v}^{(p)}\right\|_{L^{2}(\mathbb{P})}^{2}+\lambda_{0}\left\|\chi_{\lambda, v}^{(p)}\right\|_{1}^{2} \leq\left\|c_{p}\right\|_{-1}\left\|\chi_{\lambda, v}^{(p)}\right\|_{1},
$$

which leads to the estimates

$$
\sup _{\lambda \in(0,1]}\left\|\chi_{\lambda, v}^{(p)}\right\|_{1} \leq \frac{1}{\lambda_{0}}\left\|c_{p}\right\|_{-1}
$$

and

$$
\sup _{\lambda \in(0,1]} \lambda\left\|\chi_{\lambda, v}^{(p)}\right\|_{L^{2}(\mathbb{P})}^{2} \leq \frac{1}{\lambda_{0}}\left\|c_{p}\right\|_{-1}^{2} .
$$

In fact, more can be deduced: see (3.14) and (3.15) below.

Observe that

$$
\mathcal{L}^{(v)} F=L_{0} F+B F,
$$

where $L_{0}, B$ are the closures of $L_{0} F:=\left(\lambda_{0} / 2\right) \Delta F+\sum_{p=1}^{d} v_{p} D_{p} F$ and

$$
B F:=\frac{1}{2} \sum_{p, q=1}^{d} D_{p}\left[\left(a_{p q}-\lambda_{0} \Delta_{p q}\right) D_{q} F\right], \quad F \in C^{2}(\Omega),
$$

respectively. Note that

$$
\left\langle F,\left(-L_{0}\right) F\right\rangle_{\mathbb{P}} \leq\left\langle F,\left(-\mathcal{L}^{(v)}\right) F\right\rangle_{\mathbb{P}} \leq C_{*}\left\langle F,\left(-L_{0}\right) F\right\rangle_{\mathbb{P}}, \quad \forall F \in C^{2}(\Omega),
$$


for some $C_{*}$ independent of $F$. Using standard regularity properties of diffusions one can easily verify that $C^{2}(\Omega)$ is invariant under semigroups generated by any of the operators $L_{0}, L_{0}^{*}, B$ and $B^{*}$. It is therefore a common core for each of these operators (see Proposition 1.3.3 of [1]). Moreover, the operator $L_{0}$ is normal in the sense of the definition given in Appendix B. One can also easily observe that there exists $C>0$ such that

$$
\langle F, B G\rangle_{\mathbb{P}}^{2} \leq C\left\langle F,\left(-L_{0}\right) F\right\rangle_{\mathbb{P}}\left\langle G,\left(-L_{0}\right) G\right\rangle_{\mathbb{P}}, \quad \forall F, G \in C^{2}(\Omega) .
$$

As shown in Appendix B, the above conditions imply that

$$
\lim _{\lambda \rightarrow 0+} \lambda\left\|\chi_{\lambda, v}^{(p)}\right\|_{L^{2}(\mathbb{P})}^{2}=0
$$

and

$$
\chi_{v}^{(p)}:=\lim _{\lambda \rightarrow 0+} \chi_{\lambda, v}^{(p)} \quad \text { exists in } H_{1} .
$$

The latter is equivalent to the fact that $D_{q} \chi_{v}^{(p)} \in L^{2}(\mathbb{P}), q=1, \ldots, d$, and

$$
\lim _{\lambda \rightarrow 0+} D_{q} \chi_{\lambda, v}^{(p)}=D_{q} \chi_{v}^{(p)}
$$

in the $L^{2}$ norm for $q=1, \ldots, d$. Note also that in light of (3.9) we obtain

$$
\left\|\chi_{v}^{(p)}\right\|_{1} \leq \frac{1}{\lambda_{0}}\left\|c_{p}\right\|_{-1}
$$

Using (3.4) and (3.8) we can write

$$
x_{p}(t)-v_{p} t=R_{p, \lambda}(t)+\widetilde{M}_{p, \lambda}(t),
$$

where $\widetilde{M}_{p, \lambda}(t):=M_{p}(t)+N_{p, \lambda}(t)$ and $M(t)=\left(M_{1}(t), \ldots, M_{d}(t)\right)$ is given by (3.5) while

$$
N_{p, \lambda}(t):=\chi_{\lambda, v}^{(p)}\left(\zeta_{t}\right)-\chi_{\lambda, v}^{(p)}\left(\zeta_{0}\right)-\int_{0}^{t} \mathcal{L}^{(v)} \chi_{\lambda, v}^{(p)}\left(\zeta_{s}\right) d s .
$$

Note that by Itô's formula,

$$
\widetilde{M}_{p, \lambda}(t)=\int_{0}^{t}\left(\vec{e}_{p}+\nabla \chi_{\lambda, v}^{(p)}\right) \sigma\left(\zeta_{s}\right) d w(s), \quad t \geq 0 .
$$

The remainder term is

$$
R_{p, \lambda}(t):=\chi_{\lambda, v}^{(p)}\left(\zeta_{0}\right)-\chi_{\lambda, v}^{(p)}\left(\zeta_{t}\right)+\lambda \int_{0}^{t} \chi_{\lambda, v}^{(p)}\left(\zeta_{s}\right) d s .
$$

Let

$$
\widetilde{M}_{p}(t):=\int_{0}^{t}\left(\vec{e}_{p}+\nabla \chi_{v}^{(p)}\right) \sigma\left(\zeta_{s}\right) d w(s), \quad t \geq 0 .
$$


From the above and (3.19) we see that the quadratic variation of $\left(\widetilde{M}_{p, \lambda}(t)-\right.$ $\left.\widetilde{M}_{p}(t)\right)_{t \geq 0}$ equals

$$
\left\langle\widetilde{M}_{p, \lambda}-\widetilde{M}_{p}\right\rangle_{t}=\int_{0}^{t} a^{\mathrm{s}}\left(\zeta_{s}\right)\left(\nabla \chi_{\lambda, v}^{(p)}-\nabla \chi_{v}^{(p)}\right)\left(\zeta_{s}\right) \cdot\left(\nabla \chi_{\lambda, v}^{(p)}-\nabla \chi_{v}^{(p)}\right)\left(\zeta_{s}\right) d s
$$

for any $\lambda>0$. Thus, in light of (3.15) and (3.16), the martingales $\left(\widetilde{M}_{\lambda}(t)\right)_{t \geq 0}$ converge, as $\lambda \rightarrow 0+$, in the Hilbert space norm induced by the quadratic covariation, to $\widetilde{M}(t)=\left(\widetilde{M}_{1}(t), \ldots, \widetilde{M}_{d}(t)\right), t \geq 0$. As a consequence, also $R_{p, \lambda}(t) \rightarrow R_{p}(t)$ in $L^{2}(\mathbb{P})$ as $\lambda \rightarrow 0+$ for $p=1, \ldots, d$. Note that

$$
\begin{aligned}
& \frac{R_{p}(t)}{\sqrt{t}} \\
& \quad=\frac{1}{\sqrt{t}}\left[\widetilde{M}_{p, \lambda}(t)-\widetilde{M}_{p}(t)+\chi_{\lambda, v}^{(p)}\left(\zeta_{0}\right)-\chi_{\lambda, v}^{(p)}\left(\zeta_{t}\right)+\lambda \int_{0}^{t} \chi_{\lambda, v}^{(p)}\left(\zeta_{s}\right) d s\right] .
\end{aligned}
$$

Thus, using an elementary estimate $\left(\sum_{i=1}^{4} a_{i}\right)^{2} \leq 4 \sum_{i=1}^{4} a_{i}^{2}$ and (3.20), we obtain from (3.21)

$$
\begin{aligned}
\frac{1}{t}\left\langle E_{\mathbb{Q}} R_{p}^{2}(t)\right\rangle_{\mathbb{P}} \leq & 4\left\langle a^{\mathrm{s}}\left(\nabla \chi_{\lambda, v}^{(p)}-\nabla \chi_{v}^{(p)}\right), \nabla \chi_{\lambda, v}^{(p)}-\nabla \chi_{v}^{(p)}\right\rangle_{\mathbb{P}} \\
& +\frac{4}{t}\left[2+(\lambda t)^{2}\right]\left\|\chi_{\lambda, v}^{(p)}\right\|_{L^{2}(\mathbb{P})}^{2} .
\end{aligned}
$$

Substituting in the above estimate $\lambda=1 / t$ and using (3.14) and (3.16), we obtain

$$
\lim _{t \rightarrow \infty} \frac{1}{t}\left\langle E_{\mathbb{Q}} R_{p}^{2}(t)\right\rangle_{\mathbb{P}}=0 .
$$

By virtue of the ergodic theorem,

$$
\frac{1}{t}\left\langle\widetilde{M}_{p}, \widetilde{M}_{q}\right\rangle_{t}=\frac{1}{t} \int_{0}^{t} a^{\mathrm{s}}\left(\zeta_{s}\right)\left(\vec{e}_{p}+\nabla \chi_{v}^{(p)}\left(\zeta_{s}\right)\right) \cdot\left(\vec{e}_{q}+\nabla \chi_{v}^{(q)}\left(\zeta_{s}\right)\right) d s \rightarrow D_{p q}(v)
$$

as $t \rightarrow \infty$, both a.s. and in $L^{1}$ for each $p, q=1, \ldots, d$. Here,

$$
D_{p q}(v):=\left\langle a^{\mathrm{s}}\left(\vec{e}_{p}+\nabla \chi_{v}^{(p)}\right), \vec{e}_{q}+\nabla \chi_{v}^{(q)}\right\rangle_{\mathbb{P}} .
$$

Using the central limit theorem for martingales with stationary and ergodic increments (see e.g. Theorem 5.4 of [3]), we conclude that the laws of $\widetilde{M}(t) / \sqrt{t}$ converge weakly to the law of a normal random vector as $t \rightarrow \infty$. Applying the decomposition (3.18) together with (3.22), we can justify the central limit theorem for $(x(t)-v t) / \sqrt{t}$ as $t \rightarrow \infty$. The limiting law is that of a normal random vector with zero mean and with covariance matrix $D(v)=\left[D_{p q}(v)\right]$. 
4. The proof of Theorem 2.2. Suppose that $E(d k)$ is the spectral resolution corresponding to the group $\left(T_{x}\right)_{x \in \mathbb{R}^{d}}$. It is an $L^{2}(\mathbb{P})$ orthogonal projection valued Borel measure defined on $\left(\mathbb{R}^{d}, \mathcal{B}\left(\mathbb{R}^{d}\right)\right)$ such that $T_{x}=$ $\int e^{i k \cdot x} E(d k)$ for $x \in \mathbb{R}^{d}$. For a given $\phi \in L^{2}(\mathbb{P})$ one can define a numerical, finite, Borel measure $e_{\phi}(A):=\langle E(A) \phi, \phi\rangle_{\mathbb{P}}$ for $A \in \mathcal{B}\left(\mathbb{R}^{d}\right)$. It is called the spectral measure of $\phi$. In fact, the definition of the spectral measure can be extended to the elements of the spaces $H^{ \pm 1}$. Let $\mathcal{B}_{0}$ be a subfamily of subsets $A \in \mathcal{B}\left(\mathbb{R}^{d}\right)$ that are separated from 0 , i.e. $\operatorname{dist}(0, A)>0$, and bounded, i.e. there exists $R>0$ for which $A \subset B(0, R)$.

Proposition 4.1. Suppose that $\phi \in H^{1}$ or $\phi \in H^{-1}$. Then there exists a unique measure $e_{\phi}$, also called the spectral measure of $\phi$, such that $e_{\phi}(\{0\})=0$ and for any sequence $\left(\phi_{n}\right)_{n \geq 1}$ in $H_{0}^{1}$ or in $H_{0}^{-1}$ that converges to $\phi$ in the respective norm we have

$$
\lim _{n \rightarrow \infty} e_{\phi_{n}}(A)=e_{\phi}(A) \quad \text { for all } A \in \mathcal{B}_{0} .
$$

In addition, for $\phi \in H^{1}$ we have

$$
\|\phi\|_{1}^{2}=\int|k|^{2} e_{\phi}(d k)<\infty
$$

while for $\phi \in H^{-1}$,

$$
\|\phi\|_{-1}^{2}=\int \frac{e_{\phi}(d k)}{|k|^{2}}<\infty .
$$

Conversely, suppose that $\phi \in L^{2}(\mathbb{P})$ has spectral measure $e_{\phi}$ that satisfies either (4.2) or (4.3). Then it belongs to $H^{1}$ or $H^{-1}$, respectively.

Proof. The group $\left(T_{x}\right)_{x \in \mathbb{R}^{d}}$ can be extended to act as a $C_{0}$ group of unitary maps on $H^{1}$. Its spectral resolution $E^{1}(d k)$ allows us to define the spectral measure $e_{\phi}^{1}(d k):=\left(E^{1}(d k) \phi, \phi\right)_{1}$ corresponding to any $\phi \in H^{1}$. Note that for $\phi \in H_{0}^{1}$ we have

$$
e_{\phi}^{1}(d k)=|k|^{2} e_{\phi}(d k) .
$$

Indeed, we then have $T_{x} \phi=\int e^{i k \cdot x} E^{1}(d k) \phi$. Hence, for any $f \in \mathcal{S}\left(\mathbb{R}^{d}\right)$,

$$
\left\langle\int f(x) T_{x} \phi d x, \phi\right\rangle_{1}=\int \check{f}(k) e_{\phi}^{1}(d k),
$$

where $\check{f}(k):=\int e^{i k \cdot x} f(x) d x$ denotes the inverse Fourier transform of $f$. On the other hand, the left hand side of the above equality equals

$$
\sum_{p=1}^{d}\left\langle\int f(x) T_{x} D_{p} \phi d x, D_{p} \phi\right\rangle_{\mathbb{P}}=\int|k|^{2} \check{f}(k) e_{\phi}(d k) .
$$

Since the above equalities hold for all $f \in \mathcal{S}\left(\mathbb{R}^{d}\right)$ we infer that (4.4) remains valid for $\phi \in H_{0}^{1}$. We can now use (4.4) to define the spectral measure $e_{\phi}$, not necessarily finite, for any $\phi \in H^{1}$. Of course, (4.2) follows directly from 
the definition. For any $A \in \mathcal{B}\left(\mathbb{R}^{d}\right)$ we have $e_{\phi_{n}-\phi}(A) \leq \Delta^{-2}\left\|\phi_{n}-\phi\right\|_{1}^{2}$, provided that $\operatorname{dist}(0, A) \geq \Delta$. From the triangle inequality we also have $\left|e_{\phi_{n}}^{1 / 2}(A)-e_{\phi}^{1 / 2}(A)\right| \leq e_{\phi_{n}-\phi}^{1 / 2}(A)$. These two facts yield (4.1). Suppose now that the spectral measure satisfies $\int|k|^{2} e_{\phi}(d k)<\infty$. It is straightforward to check that this condition implies the existence of $D_{p} \phi$, in fact $D_{p} \phi=i \int k_{p} E(d k)$ for $p=1, \ldots, d$. Thus, $\phi$ belongs to $H^{1}$ (see (3.6)).

The group $\left(T_{x}\right)_{x \in \mathbb{R}^{d}}$ also extends to a strongly continuous, unitary group on $H^{-1}$, which allows one to define the spectral resolution $E^{-1}(d k)$, which leads to the definition of the spectral measure $e_{\phi}^{-1}(d k)$. For $\phi \in H_{0}^{-1}$ we can show, using duality, that $e_{\phi}^{-1}(d k)=|k|^{-2} e_{\phi}(d k)$. This equality allows extending the definition of $e_{\phi}(d k)$ to all $\phi \in H^{-1}$. The remaining part of the argument is quite analogous to the $H^{1}$ case and we leave it to the reader.

For $\phi \in H_{0}^{1}$ we let $A \phi:=v \cdot \nabla \phi \in L^{2}(\mathbb{P})$. Since $\|A \phi\|_{L^{2}(\mathbb{P})} \leq|v|_{\ell^{2}}\|\phi\|_{1}$, where $|v|_{\ell^{2}}:=\left(\sum_{i=1}^{d} v_{i}^{2}\right)^{1 / 2}$, the operator $A$ can be extended to a bounded operator from $H^{1}$ to $L^{2}(\mathbb{P})$. Note also that

$$
e_{A \phi}(d k)=\frac{(v \cdot k)^{2}}{|k|^{2}} e_{\phi}^{1}(d k) .
$$

Proposition 4.2. Suppose that $\phi \in H^{1}$ and $A \phi \in H^{-1}$. Then

$$
{ }_{-1}\langle A \phi, \phi\rangle_{1}=0 .
$$

Here ${ }_{-1}\langle\cdot, \cdot\rangle_{1}$ denotes the duality pairing between the spaces $H^{1}$ and $H^{-1}$.

Proof. Since $\nabla$ is a generator of a unitary group on $L^{2}(\mathbb{P})$ each of its components is anti-self-adjoint. Consequently, we obtain

$$
\langle A \phi, \phi\rangle_{\mathbb{P}}=0, \quad \forall \phi \in H_{0}^{1} .
$$

Suppose that $\phi \in H^{1}$. Let $\phi_{n}:=\int_{[|k| \geq 1 / n]} E^{1}(d k) \phi$. We have $\lim _{n \rightarrow \infty} \phi_{n}=\phi$ in $H^{1}$ and $\left(\phi_{n}\right)_{n \geq 1} \subset H_{0}^{1}$, and by virtue of (4.7),

$$
{ }_{-1}\left\langle A \phi_{n}, \phi_{n}\right\rangle_{1}=0, \quad \forall n \geq 1 \text {. }
$$

Observe also that $A \phi_{n} \rightarrow A \phi$ in $L^{2}(\mathbb{P})$. We have

$$
\begin{aligned}
\left\|A \phi_{n}\right\|_{-1}^{2} & =\int \frac{e_{A \phi_{n}}(d k)}{|k|^{2}} \stackrel{(4.5)}{=} \int \frac{(k \cdot v)^{2}}{|k|^{4}} e_{\phi_{n}}^{1}(d k) \\
& =\int_{[|k| \geq 1 / n]} \frac{(k \cdot v)^{2}}{|k|^{4}} e_{\phi}^{1}(d k) \leq \int \frac{(k \cdot v)^{2}}{|k|^{4}} e_{\phi}^{1}(d k)=\|A \phi\|_{-1}^{2}<\infty .
\end{aligned}
$$

Hence, some subsequence of $\left(A \phi_{n}\right)_{n \geq 1}$ converges weakly in $H^{-1}$. Since it converges in $L^{2}$ to $A \phi$ its $H^{-1}$-weak limit must be equal to $A \phi$ as well. Knowing that $\left(\phi_{n}\right)_{n \geq 1}$ converges strongly in $H^{1}$ we obtain

$$
0=\lim _{n \rightarrow \infty}{ }_{-1}\left\langle A \phi_{n}, \phi_{n}\right\rangle_{1}={ }_{-1}\langle A \phi, \phi\rangle_{1} .
$$


Directly from the definition (see (3.11)), one can observe that $B$ extends to a bounded operator from $H^{1}$ to $H^{-1}$. Furthermore, directly from the definition, we have ${ }_{-1}\langle(-B) \phi, \phi\rangle_{1} \geq 0$ for all $\phi \in H^{1}$.

Proposition 4.3. Suppose that $\phi \in H^{1}$ is such that $A \phi \in H^{-1}$ and

$$
-\left(\frac{\lambda_{0}}{2} \Delta+A\right) \phi-B \phi=c_{p} \text {. }
$$

Then $\phi=\chi_{v}^{(p)}$.

Proof. Observe that, upon letting $\lambda \rightarrow 0+$, it follows from (3.8) that $A \chi_{v}^{(p)} \in H^{-1}$ and $\chi_{v}^{(p)}$ satisfies (4.10). We can therefore write

$$
-\left(\frac{\lambda_{0}}{2} \Delta+A\right)\left(\phi-\chi_{v}^{(p)}\right)-B\left(\phi-\chi_{v}^{(p)}\right)=0 .
$$

Testing both sides of the above equation with $\phi-\chi_{v}^{(p)}$ and using Proposition 4.2 we conclude that $\left\|\phi-\chi_{v}^{(p)}\right\|_{1}^{2}=0$.

THEOREM 4.4. The function $v \mapsto \chi_{v}^{(p)} \in H^{1}$ is strongly continuous.

Proof. Suppose $v_{n} \rightarrow v_{0}$. From (3.17) we have $\left\|\chi_{v_{n}}^{(p)}\right\|_{1} \leq C$ with $C$ independent of $n$. In addition, since each $\chi_{v_{n}}^{(p)}$ satisfies (4.10) we conclude that also $\left\|A \chi_{v_{n}}^{(p)}\right\|_{-1} \leq C$ for a certain constant independent of $n$. Both of these sequences are therefore weakly relatively compact in the respective spaces.

We show that $\left(\chi_{v_{n}}^{(p)}\right)_{n \geq 1}$ is weakly convergent in $H^{1}$. Note that since each $\chi_{v_{n}}^{(p)}$ satisfies (4.10) this fact necessarily implies that $\left(A \chi_{v_{n}}^{(p)}\right)_{n \geq 1}$ converges $H^{-1}$-weakly. It suffices therefore to show that $\left(\chi_{v_{n}}^{(p)}\right)_{n \geq 1}$ has a unique weak limiting point. So suppose that $\chi_{*}^{(p)}:=w-\lim _{n \rightarrow \infty} \chi_{v_{n}}^{(p)}$ in $H^{1}$. Then also $\left(A \chi_{v_{n}}^{(p)}\right)_{n \geq 1}$ converges $H^{-1}$-weakly to $A \chi_{*}^{(p)}$. Since $A \chi_{*}^{(p)} \in H^{-1}$ we can claim that $\chi_{*}^{(p)}$ satisfies (4.10) with $v=v_{0}$. Thus, according to Proposition 4.3, it equals $\chi_{v_{0}}^{(p)}$ and the uniqueness claim follows.

We show that convergence is in fact strong in the $H^{1}$ norm. We have

$$
-\left(\frac{\lambda_{0}}{2} \Delta+A\right) \chi_{v_{n}}^{(p)}-B \chi_{v_{n}}^{(p)}=c_{p}, \quad \forall n \geq 0 .
$$

After testing both sides with $\chi_{v_{n}}^{(p)}$, we obtain

$$
-_{-1}\left\langle\left(\frac{\lambda_{0}}{2} \Delta+B\right) \chi_{v_{n}}^{(p)}, \chi_{v_{n}}^{(p)}\right\rangle_{1}={ }_{-1}\left\langle c_{p}, \chi_{v_{n}}^{(p)}\right\rangle_{1}, \quad \forall n \geq 0 .
$$


Taking the limits on both sides we obtain

$$
\begin{aligned}
\frac{1}{2} \lim _{n \rightarrow \infty} \sum_{k, l=1}^{d}\left\langle a_{k l}^{\mathrm{s}} D_{k} \chi_{v_{n}}^{(p)}, D_{l} \chi_{v_{n}}^{(p)}\right\rangle_{\mathbb{P}} & ={ }_{-1}\left\langle c_{p}, \chi_{v_{0}}^{(p)}\right\rangle_{1} \\
& =\frac{1}{2} \sum_{k, l=1}^{d}\left\langle a_{k l}^{\mathrm{s}} D_{k} \chi_{v_{0}}^{(p)}, D_{l} \chi_{v_{0}}^{(p)}\right\rangle_{\mathbb{P}}
\end{aligned}
$$

Combining this fact with the already proven weak convergence we conclude that

$$
\begin{aligned}
0 & =\frac{1}{2} \lim _{n \rightarrow \infty} \sum_{k, l=1}^{d}\left\langle a_{k l}^{\mathrm{S}}\left(D_{k} \chi_{v_{n}}^{(p)}-D_{k} \chi_{v_{0}}^{(p)}\right), D_{l} \chi_{v_{n}}^{(p)}-D_{l} \chi_{v_{0}}^{(p)}\right\rangle_{\mathbb{P}} \\
& \geq \lim _{n \rightarrow \infty} \frac{\lambda_{0}}{2}\left\|\chi_{v_{n}}^{(p)}-\chi_{v_{0}}^{(p)}\right\|_{1}^{2} .
\end{aligned}
$$

From the continuity of the corrector and (3.23) we conclude that the mapping $v \mapsto D(v)$ is continuous.

5. One-dimensional example. Assume that $d=1$ and, with no loss of generality, that $v>0$. The diffusivity coefficient is then a positive scalar field $a(x ; \omega):=a\left(\tau_{x} \omega\right), x \in \mathbb{R}^{d}$, with $a(\omega) \geq \lambda_{0} \mathbb{P}$-a.s. We suppose that the laws of $(a(x))_{x \in \mathbb{R}}$ and of $(a(-x))_{x \in \mathbb{R}}$ are identical.

In this case the cell problem reads

$$
-\frac{1}{2} D\left(a D \chi_{v}\right)-v D \chi_{v}=\frac{1}{2} D a .
$$

We claim that if $v \neq 0$ the above equation has a solution in $H_{0}^{1}$. From (5.1) and ergodicity of the group of spatial shifts it follows that there exists a deterministic constant $C_{v}$ such that

$$
a D \chi_{v}+2 v \chi_{v}+a=C_{v} .
$$

Let $\chi_{v}(x ; \omega):=\chi_{v}\left(\tau_{x} \omega\right)$. Since $\chi_{v}^{\prime}(x ; \omega)=D \chi_{v}\left(\tau_{x} \omega\right)$ we can rewrite $(5.2)$ as the first order linear o.d.e.

$$
a(x) \chi_{v}^{\prime}(x)+2 v \chi_{v}(x)=C_{v}-a(x) .
$$

It can be solved explicitly and we obtain

$$
\begin{aligned}
\chi_{v}(x ; \omega)= & C_{1}(\omega) \exp \left\{-2 v \int_{0}^{x} \frac{d y}{a(y ; \omega)}\right\} \\
& +\int_{0}^{x}\left[\frac{C_{v}}{a(y ; \omega)}-1\right] \exp \left\{-2 v \int_{y}^{x} \frac{d z}{a(z ; \omega)}\right\} d y,
\end{aligned}
$$

where $C_{1}(\omega)$ is a random variable. We assume that for any $\Delta>0$ there exists a random variable $A(\omega)>0$ such that $\left|C_{1}\left(\tau_{x} \omega\right)\right| \leq A(\omega) e^{\Delta|x|}$. Then 


$$
\begin{aligned}
\chi_{v}(\omega)=\chi_{v}\left(x ; \tau_{-x} \omega\right) & =C_{1}\left(\tau_{-x} \omega\right) \exp \left\{-2 v \int_{0}^{x} \frac{d y}{a\left(y ; \tau_{-x} \omega\right)}\right\} \\
& +\int_{0}^{x}\left[\frac{C_{v}}{a\left(y ; \tau_{-x} \omega\right)}-1\right] \exp \left\{-2 v \int_{y}^{x} \frac{d z}{a\left(z ; \tau_{-x} \omega\right)}\right\} d y .
\end{aligned}
$$

Using the fact that $a(\omega)$ is deterministically bounded from above and letting $x \rightarrow \infty$ we conclude that the first term on the right hand side of (5.5) has to vanish. On the other hand, in the second term we can change variables $y^{\prime}:=y-x$, use the identity $a\left(z ; \tau_{-x} \omega\right)=a(z-x ; \omega)$ and then let $x \rightarrow \infty$. We conclude in this way that the corrector must be of the form

$$
\chi_{v}(\omega)=\int_{-\infty}^{0}\left[\frac{C_{v}}{a(y ; \omega)}-1\right] \exp \left\{-2 v \int_{y}^{0} \frac{d z}{a(z ; \omega)}\right\} d y .
$$

To determine $C_{v}$ we use the condition that $\chi_{v}$ is, in particular, of zero mean. Thus, taking into account that $(a(y))_{y \in \mathbb{R}}$ and $(a(-y))_{y \in \mathbb{R}}$ have the same laws we obtain

$$
C_{v}=\frac{\left\langle\int_{0}^{\infty} \exp \left\{-2 v \int_{0}^{y} a^{-1}(z) d z\right\} d y\right\rangle_{\mathbb{P}}}{\left\langle\int_{0}^{\infty} a^{-1}(y) \exp \left\{-2 v \int_{0}^{y} a^{-1}(z) d z\right\} d y\right\rangle_{\mathbb{P}}} .
$$

One can verify by a direct calculation that $\chi_{v}$ given by (5.5) together with (5.6) is indeed a solution to the cell problem (5.1).

According to (3.23) the effective diffusivity coefficient equals

$$
D(v)=\left\langle a\left(1+D \chi_{v}\right)\left(1+D \chi_{v}\right)\right\rangle_{\mathbb{P}} \stackrel{(5.2)}{=}\left\langle\left(C_{v}-2 v \chi_{v}\right)\left(1+D \chi_{v}\right)\right\rangle_{\mathbb{P}}=C_{v} .
$$

In the last equality we have used the fact that $\int \chi_{v} d \mathbb{P}=0$ and $\int D \psi d \mathbb{P}=0$ for any $\psi$ and $\chi_{v} D \chi_{v}=\frac{1}{2} D \chi_{v}^{2}$.

Let $b:=a^{-1}-\left\langle a^{-1}\right\rangle_{\mathbb{P}}$. Denote by $R(y)$ the covariance function of the random field $b(y ; \omega):=b\left(\tau_{y} \omega\right)$. We shall assume that the field has good mixing properties, i.e. in particular that $v^{1 / 2} \int_{0}^{y / v} b(z) d z$ converges weakly, as $v \rightarrow 0$, to a zero mean Brownian motion with the variance

$$
\sigma^{2}:=2 \int_{0}^{\infty} R(y) d y<\infty .
$$

Then, of course, $v^{s} \int_{0}^{y / v} b(z) d z \rightarrow 0$ in probability when $s>1 / 2$.

Set

$$
\begin{aligned}
& F(v):=v\left\langle\int_{0}^{\infty} \exp \left\{-2 v \int_{0}^{y} a^{-1}(z) d z\right\} d y\right\rangle_{\mathbb{P}}, \\
& G(v):=v\left\langle\int_{0}^{\infty} a^{-1}(y) \exp \left\{-2 v \int_{0}^{y} a^{-1}(z) d z\right\} d y\right\rangle_{\mathbb{P}} .
\end{aligned}
$$


We have $C_{v}=F(v) / G(v)$ and, after a change of variables $y:=v y$, we obtain

$$
\begin{aligned}
F(v) & =\int_{0}^{\infty} e^{-2 y\left\langle a^{-1}\right\rangle_{\mathbb{P}}}\left\langle\exp \left\{-2 v \int_{0}^{y / v} b(z) d z\right\}\right\rangle_{\mathbb{P}} d y \\
& =\int_{0}^{\infty} e^{-2 y\left\langle a^{-1}\right\rangle_{\mathbb{P}}}\left\langle\left[1-2 v \int_{0}^{y / v} b(z) d z+2 v^{2}\left(\int_{0}^{y / v} b(z) d z\right)^{2}+o\left(v^{2}\right)\right]\right\rangle_{\mathbb{P}} d y \\
& =\int_{0}^{\infty} e^{-2 y\left\langle a^{-1}\right\rangle_{\mathbb{P}}}\left(1+2 v \sigma^{2} y+o(v)\right) d y=\frac{1}{2\left\langle a^{-1}\right\rangle_{\mathbb{P}}}+\frac{v \sigma^{2}}{2\left\langle a^{-1}\right\rangle_{\mathbb{P}}^{2}}+o(v) .
\end{aligned}
$$

From isotropy of the diffusivity we conclude that

$$
F(v)=\frac{1}{2\left\langle a^{-1}\right\rangle_{\mathbb{P}}}+\frac{|v| \sigma^{2}}{2\left\langle a^{-1}\right\rangle_{\mathbb{P}}^{2}}+o(v), \quad \forall v \neq 0 .
$$

Analogously we can write

$$
\begin{aligned}
G(v) & =v \int_{0}^{\infty}\left\langle a^{-1}(y) \exp \left\{-2 v \int_{0}^{y} a^{-1}(z) d z\right\}\right\rangle_{\mathbb{P}} d y \\
& =-\frac{1}{2}\left\langle\int_{0}^{\infty} \frac{d}{d y} \exp \left\{-2 v \int_{0}^{y} a^{-1}(z) d z\right\} d y\right\rangle_{\mathbb{P}}=\frac{1}{2} .
\end{aligned}
$$

Therefore we have

$$
D(v)=\frac{1}{\left\langle a^{-1}\right\rangle_{\mathbb{P}}}+\frac{|v| \sigma^{2}}{\left\langle a^{-1}\right\rangle_{\mathbb{P}}^{2}}+o(v), \quad \forall v \neq 0 .
$$

The function is continuous at 0 but is not of class $C^{1}$.

\section{The proof of Theorem 2.6}

Proposition 6.1. The tensor valued mapping $v \mapsto D(v)$ is isotropic.

Proof. Let $g \in O(d)$ and let $x^{(v)}(t)$ denote the diffusion with drift $v$ given by

$$
d x^{(v)}(t)=a^{1 / 2}\left(x^{(v)}(t)\right) d w(t)+\left[\frac{1}{2} \nabla \cdot a\left(x^{(v)}(t)\right)+v\right] d t,
$$

where $(w(t))_{t \geq 0}$ is a standard $d$-dimensional Brownian motion. We show that the laws of $x^{(g v)}(t)$ and $g x^{(v)}(t)$ are the same. This, in turn, implies the isotropy of $v \mapsto D(v)$. Isotropy of the field $(a(x))_{x \in \mathbb{R}^{d}}$ (see Definition 2.3) implies that the joint laws of the fields $\left(a(g x), a^{1 / 2}(g x),(\nabla \cdot a)(g x) g\right)_{x \in \mathbb{R}^{d}}$ and of $\left(g a(x) g^{T}, g a^{1 / 2}(x) g^{T}, \nabla \cdot a(x)\right)_{x \in \mathbb{R}^{d}}$ are the same. The law of $\left(g x^{(v)}(t)\right)_{t \geq 0}$ is identical with that of the solution to

$$
d y(t)=a^{1 / 2}(y(t)) g d w(t)+\left[\frac{1}{2} \nabla \cdot a(y(t))+g v\right] d t, \quad y(0)=0 .
$$

Since $(g w(t))_{t \geq 0}$ is another standard Brownian motion we conclude that the law of $(y(t))_{t \geq 0}$ coincides with that of $\left(x^{(g v)}(t)\right)_{t \geq 0}$. 
We proceed with the proof of Theorem 2.6. For $\theta \in \mathbb{R}$ and fixed $v \neq 0$ the corrector $\chi_{\theta v}^{(p)} \in H^{1}$ satisfies

$$
-\mathcal{L}_{\theta} \chi_{\theta v}^{(p)}=c_{p},
$$

where $\mathcal{L}_{\theta}:=\left(\lambda_{0} / 2\right) \Delta+\theta A+B$. We show that $\theta \mapsto \chi_{\theta v}^{(p)}$ is differentiable in $H^{1}$ at any $\theta \neq 0$. Let

$$
\delta \chi^{(p)}(\theta, h):=\frac{\chi_{(\theta+h) v}^{(p)}-\chi_{\theta v}^{(p)}}{h} .
$$

We have

$$
-\mathcal{L}_{\theta} \delta \chi^{(p)}(\theta, h)=A \chi_{(\theta+h) v}^{(p)} .
$$

The key observation is the following.

Lemma 6.2. For each $\theta \neq 0$ the mapping $h \mapsto A \chi_{(\theta+h) v}^{(p)}$ is continuous at 0 in the $H^{-1}$ norm.

Proof. By Theorem 4.4 the mapping $h \mapsto \chi_{(\theta+h) v}^{(p)}$ is continuous at 0 in the $H^{1}$ norm. This implies that $h \mapsto \mathcal{L}_{\theta} \chi_{(\theta+h) v}^{(p)}$ is continuous at 0 in the $H^{-1}$ norm. From (6.2) we deduce that

$$
A \chi_{(\theta+h) v}^{(p)}=-(\theta+h)^{-1}\left(c_{p}+\mathcal{L}_{0} \chi_{(\theta+h) v}^{(p)}\right)
$$

and the conclusion of the lemma follows easily.

The convergence of $\delta \chi^{(p)}(\theta, h)$ in the $H^{1}$ norm, as $h \rightarrow 0$, now follows easily from the argument similar to the one used in the proof of Theorem 4.4. We then find that the limit $\frac{d}{d \theta} \chi_{\theta v}^{(p)}$ satisfies

$$
-\mathcal{L}_{\theta}\left(\frac{d \chi_{\theta v}^{(p)}}{d \theta}\right)=A \chi_{\theta v}^{(p)}
$$

One can use again the aforementioned argument to conclude that the function $\theta \mapsto \frac{d}{d \theta} \chi_{\theta v}^{(p)}$ is $H^{1}$-continuous, except possibly at $\theta=0$. By recursion this claim can be extended to higher order derivatives $\frac{d^{n}}{d \theta^{n}} \chi_{\theta v}^{(p)}$ for all $n \geq 1$.

$C^{\infty}$-smoothness of the corrector along the direction of a vector $v$ implies of course $C^{\infty}$-differentiability of $v \mapsto D(v)$ with respect to the radial variable $|v|$. Its differentiability with respect to the angular variable $\widehat{v}:=v /|v|$ follows from isotropy of the tensor (see Proposition 2.4). Thus, the conclusion of Theorem 2.6 follows.

REMARK 6.3. Observe that the above argument relies on isotropy of the diffusivity field $x \mapsto a(x)$. If we tried to prove differentiability of the mapping $v \mapsto \chi_{v}^{(p)}$ in a direction $\ell \perp v$, the approximation scheme (6.3) would break down. Note that then $\delta_{\ell} \chi^{(p)}(h):=h^{-1}\left[\chi_{v+h \ell}^{(p)}-\chi_{v}^{(p)}\right]$ satisfies 
$-\mathcal{L}_{1} \delta_{\ell} \chi^{(p)}(h)=A \chi_{v+h \ell}^{(p)}$. The function $h \mapsto A \chi_{v+h \ell}^{(p)}$ is continuous in the $L^{2}(\mathbb{P})$ norm, by virtue of Theorem 4.4. One cannot, though, use the cell problem (4.10) to bootstrap the continuity to the $H^{-1}$ norm. Thus, it is not possible to apply the argument of Theorem 4.4 to obtain the desired differentiability property in the $\ell$ direction.

A. The proof of Proposition 2.4. Let $g \vec{e}_{1}=\vec{e}_{1}$ and $g \vec{e}_{i}=-\vec{e}_{i}$ for $i=2, \ldots, d$. Observe that for any $t \in \mathbb{R}$ and $i \geq 2$ we have

$$
\begin{aligned}
B_{1 i}\left(t \vec{e}_{1}\right) & =B\left(t \vec{e}_{1}\right) \vec{e}_{i} \cdot \vec{e}_{1}=-B\left(g\left(t \vec{e}_{1}\right)\right) g^{T} \vec{e}_{i} \cdot g^{T} \vec{e}_{1} \\
& =-B\left(t \vec{e}_{1}\right) \vec{e}_{i} \cdot \vec{e}_{1}=-B_{1 i}\left(t \vec{e}_{1}\right) .
\end{aligned}
$$

Suppose that $i \neq j$ and $j \neq 1$. Consider $g$ such that $g \vec{e}_{k}=\vec{e}_{k}$ for $k \neq j$ and $g \vec{e}_{j}=-\vec{e}_{j}$. We show by a similar argument that $B_{i j}\left(t \vec{e}_{1}\right)=0$. The matrix $B\left(t \vec{e}_{1}\right)$ is therefore diagonal. Suppose that $\sigma$ is a permutation of $\{2, \ldots, d\}$. We let $g \vec{e}_{1}=\vec{e}_{1}$ and $g \vec{e}_{i}=\vec{e}_{\sigma(i)}$ for $i=2, \ldots, d$. Note that for any $i \geq 2$,

$$
\begin{aligned}
B_{i i}\left(t \vec{e}_{1}\right) & =B\left(t \vec{e}_{1}\right) \vec{e}_{i} \cdot \vec{e}_{i}=B\left(g\left(t \vec{e}_{1}\right)\right) g^{T} \vec{e}_{i} \cdot g^{T} \vec{e}_{i} \\
& =B\left(t \vec{e}_{1}\right) \vec{e}_{\sigma^{-1}(i)} \cdot \vec{e}_{\sigma^{-1}(i)}=B_{\sigma^{-1}(i), \sigma^{-1}(i)}\left(t \vec{e}_{1}\right) .
\end{aligned}
$$

Thus $B_{i i}\left(t \vec{e}_{1}\right)=u(t)$ for $i=2, \ldots, d$. We also let $v(t):=B_{11}\left(t \vec{e}_{1}\right)$.

Let $g_{x} \in O(d)$ be such that $g_{x} \vec{e}_{1}=\widehat{x}:=|x|^{-1} x$ and $g_{x} \vec{e}_{i}=\vec{f}_{i-1}$ for $i=2, \ldots, d$, where $\vec{f}_{1}, \ldots, \vec{f}_{d-1}$ is an orthonormal system satisfying $\vec{f}_{i} \cdot \widehat{x}=0$ for $i=1, \ldots, d-1$. We can then write

$$
B(x)=B\left(|x| g_{x} \vec{e}_{1}\right)=g_{x} B\left(|x| \vec{e}_{1}\right) g_{x}^{T}=u(|x|) \operatorname{Id}+[v(|x|)-u(|x|)] \widehat{x} \otimes \widehat{x} .
$$

B. The proof of (3.14) and (3.15). Recall the decomposition (3.10) of the operator $\mathcal{L}^{(v)}$. From $(3.12)$ there exist constants $0<c_{*}<C_{*}<\infty$ such that

$$
c_{*}\|F\|_{1}^{2} \leq\left\langle F,\left(-L_{0}\right) F\right\rangle_{\mathbb{P}} \leq C_{*}\|F\|_{1}^{2},
$$

for every $F \in C^{2}(\Omega)$. In view of (B.1) and the sector condition (see (3.13)), $B$ is a bounded mapping from $H^{1}$ to $H^{-1}$, i.e. there exists a constant $C_{B}>0$ such that

$$
\|B F\|_{-1} \leq C_{B}\|F\|_{1} \quad \text { for all } F \in C^{2}(\Omega) .
$$

Indeed, by definition of the $H^{-1}$ norm, the sector condition (3.13) and the bound (B.1),

$$
\|B F\|_{-1}=\sup _{\|G\|_{1}=1}\langle B F, G\rangle_{\mathbb{P}} \leq C_{0}\|F\|_{1}
$$

for some $C_{0}>0$ and all $F \in H^{1}$. According to the results in Section 1.2 of [7], to prove (3.14) and (3.15) it suffices to show that

$$
\sup _{0<\lambda \leq 1}\left\|\mathcal{L}^{(v)} \chi_{\lambda, v}^{(p)}\right\|_{-1}<\infty
$$


The closure of $L_{0}: C^{2}(\Omega) \rightarrow L^{2}(\mathbb{P})$ is normal, i.e. the operators

$$
S_{0}:=\frac{1}{2}\left(L_{0}+L_{0}^{*}\right)=\lambda_{0} \Delta \quad \text { and } \quad i A_{0}:=\frac{i}{2}\left(L_{0}-L_{0}^{*}\right)=i \sum_{p=1}^{d} v_{p} D_{p},
$$

defined on $C^{2}(\Omega)$, are essentially self-adjoint and their spectral resolutions commute. Note that the spectrum of $L_{0}$ is contained in the complex half plane $\Re \lambda \leq 0$. For given $\lambda>0$ and $V \in H^{-1}$ let $F_{\lambda}$ be the solution of the resolvent equation $\left(\lambda-L_{0}\right) F_{\lambda}=V$. Using the spectral resolution of $L_{0}$ we can write

$$
F_{\lambda}=\int_{0}^{\infty} \int_{-\infty}^{\infty} \frac{1}{\lambda+\varphi+i \tau} E(d \varphi, d \tau) V
$$

where $E(d \varphi, d \tau)$ is the spectral resolution of the identity which corresponds to the normal operator $L_{0}$. Since $V \in H^{-1}$ we obtain, thanks to (B.1),

$$
\int_{0}^{\infty} \int_{-\infty}^{\infty} \frac{1}{\varphi} e_{V}(d \varphi, d \tau) \leq \frac{1}{c_{*}}\|V\|_{-1}^{2}<\infty
$$

where $e_{V}(d \varphi, d \tau)$ stands for the spectral measure of $V$, i.e. $e_{V}(d \varphi, d \tau)=$ $\langle E(d \varphi, d \tau) V, V\rangle_{\mathbb{P}}$. Hence, using again (B.1),

$$
\left\|L_{0} F_{\lambda}\right\|_{-1}^{2} \leq C_{*} \int_{0}^{\infty} \int_{-\infty}^{\infty}\left|\frac{\varphi+i \tau}{\lambda+\varphi+i \tau}\right|^{2} \frac{e_{V}(d \varphi, d \tau)}{\varphi} \leq \frac{C_{*}}{c_{*}}\|V\|_{-1}^{2} .
$$

Recall that $\chi_{\lambda, v}^{(p)}$ is the solution of the full resolvent equation $(\lambda-L) \chi_{\lambda, v}^{(p)}=c_{p}$. Since $L=L_{0}+B$, we may rewrite the resolvent equation as

$$
\left(\lambda-L_{0}\right) \chi_{\lambda, v}^{(p)}=c_{p}+B \chi_{\lambda, v}^{(p)}
$$

By (B.4), with $c_{p}+B \chi_{\lambda, v}^{(p)}$ in place of $V$, we obtain

$$
\begin{aligned}
\sup _{0<\lambda \leq 1}\left\|\mathcal{L}^{(v)} \chi_{\lambda, v}^{(p)}\right\|_{-1} & \leq \sup _{0<\lambda \leq 1}\left\|L_{0} \chi_{\lambda, v}^{(p)}\right\|_{-1}+\sup _{0<\lambda \leq 1}\left\|B \chi_{\lambda, v}^{(p)}\right\|_{-1} \\
& \leq\left(\frac{C_{*}}{c_{*}}\right)^{1 / 2} \sup _{0<\lambda \leq 1}\left\|c_{p}+B \chi_{\lambda, v}^{(p)}\right\|_{-1}+\sup _{0<\lambda \leq 1}\left\|B \chi_{\lambda, v}^{(p)}\right\|_{-1} .
\end{aligned}
$$

By (B.2), the previous expression is bounded above by

$$
\begin{aligned}
\left(\frac{C_{*}}{c_{*}}\right)^{1 / 2}\left\|c_{p}\right\|_{-1}+ & {\left[1+\left(\frac{C_{*}}{c_{*}}\right)^{1 / 2}\right] \sup _{0<\lambda \leq 1}\left\|B \chi_{\lambda, v}^{(p)}\right\|_{-1} } \\
\leq & \left(\frac{C_{*}}{c_{*}}\right)^{1 / 2}\left\|c_{p}\right\|_{-1}+C_{B}\left[1+\left(\frac{C_{*}}{c_{*}}\right)^{1 / 2}\right] \sup _{0<\lambda \leq 1}\left\|\chi_{\lambda, v}^{(p)}\right\|_{1} .
\end{aligned}
$$

By (3.9) this sum is less than or equal to $C\left\|c_{p}\right\|_{-1}$ for some $C>0$, thus (B.3) follows. 


\section{References}

[1] S. Ethier and T. Kurtz, Markov Processes, Wiley, New York, 1986.

[2] W. Feller, An Introduction to Probability Theory and its Applications, Vol. II, 3rd ed., Wiley, 1971.

[3] I. S. Helland, Central limit theorems for martingales with discrete or continuous time, Scand. J. Statist. 9 (1982), 79-94.

[4] T. Komorowski, C. Landim and S. Olla, Fluctuations in Markov processes, preprint, http://w3.impa.br/ ${ }^{\sim}$ landim/notas.html.

[5] S. M. Kozlov, Averaging of random operators, Mat. Sb. 109 (1979), 182-202 (in Russian).

[6] E. Nieznaj, Central limit theorem for diffusion processes in an anisotropic random environment, Bull. Polish Acad. Sci. Math. 53 (2005), 187-205.

[7] S. Olla, Central limit theorems for tagged particles and diffusions in random environment, in: Milieux Aléatoires, Panoramas et Synthèses 12, Soc. Math. France, 2001, 75-100.

[8] —, Homogenization of diffusion processes in random fields, Centre de Mathématiques Appliquées, Ecole Polytechnique, Palaiseau, 1994, http://www.cmap.polytechnique.fr $/ \sim$ olla/lho.ps.

[9] H. Osada, Homogenization of diffusion processes with random stationary coefficients, in: Proc. 4-th Japan-USSR Symposium on Probability Theory, Lecture Notes in Math. 1021, Springer, 1982, 507-517.

[10] G. C. Papanicolaou and S. R. S. Varadhan, Boundary value problems with rapidly oscillating random coefficients, in: J. Fritz and J. L. Lebowitz (eds.), Random Fields, Colloq. Math. Soc. János Bolyai 27, North-Holland, 1982, 835-873.

M. Cudna

Institute of Mathematics

UMCS

Pl. Marii Curie-Skłodowskiej 1

20-031 Lublin, Poland

E-mail: mcudna@hektor.umcs.lublin.pl
T. Komorowski Institute of Mathematics UMCS

Pl. Marii Curie-Skłodowskiej 1 20-031 Lublin, Poland E-mail: komorow@hektor.umcs.lublin.pl and

Institute of Mathematics Polish Academy of Sciences Sniadeckich 8

00-956 Warszawa, Poland http://hektor.umcs.lublin.pl $/{ }^{\sim}$ komorow 